Johannes Kolmar ORCID iD: 0000-0001-9870-074X

Frank Baganz ORCID iD: 0000-0001-5589-6869

Research Article

\title{
Improving product specificity of whole-cell alkane oxidation in non-conventional media: a multivariate analysis approach
}

Johannes F Kolmar ${ }^{1}$

Oliver Thum ${ }^{2}$

Frank Baganz ${ }^{1,3}$

${ }^{1}$ Advanced Centre for Biochemical Engineering, Department of Biochemical Engineering, University College London, Bernard Katz Building, Gordon Street, London WC1H 0AH, United Kingdom

${ }^{2}$ Evonik Creavis GmbH, Paul-Baumann-Straße 1, 45772 Marl, Germany

${ }^{3}$ Corresponding author: Frank Baganz, Advanced Centre for Biochemical Engineering, Department of Biochemical Engineering, University College London, Bernard Katz Building, Gordon Street, London WC1H 0AH, United Kingdom

Email: f.baganz@ucl.ac.uk

ORCID IDs:

JF Kolmar: 0000-0001-9870-074X

O Thum: 0000-0002-2568-1450

F Baganz: 0000-0001-5589-6869

Keywords: Alkane monooxygenase, High-throughput, Multivariate data analysis, Nonconventional media, Whole-cell bio-oxidation

This article has been accepted for publication and undergone full peer review but has not been through the copyediting, typesetting, pagination and proofreading process, which may lead to differences between this version and the Version of Record. Please cite this article as doi: 10.1002/biot.201800581.

This article is protected by copyright. All rights reserved. 


\begin{abstract}
Two-liquid phase reaction media have long been used in bioconversions to supply or remove hydrophobic organic reaction substrates and products to reduce inhibitory and toxic effects on biocatalysts. In case of the terminal oxyfunctionalisation of linear alkanes by the AlkBGT monooxygenase the excess alkane substrate is often used as a second phase to extract the alcohol, aldehyde and acid products. However, the selection of other carrier phases or surfactants is complex due to the large amount of parameters that are involved, such as: biocompatibility, substrate bioavailability and product extraction selectivity. This study combines systematic high-throughput screening with chemometrics to correlate physicochemical parameters of a range of co-solvents to product specificity and yield using a multivariate regression model.
\end{abstract}

Partial least square regression showed that the defining factor for product specificity are the solubility properties of reaction substrate and product in the co-solvent, as measured by Hansen solubility parameters. Thus the polarity of co-solvents determines the accumulation of either alcohol or acid products. Whereas usually the acid product accumulates during the reaction, by choosing a more polar co-solvent the 1-alcohol product can be accumulated. Especially with Tergitol as co-solvent, a 3.2 fold improvement in 1-octanol yield to $18.3 \mathrm{mmol} \mathrm{l}^{-1}$ was achieved relative to the control reaction without co-solvents.

\title{
Graphical Abstract
}

Two-liquid phase reaction media have long been used in bioconversions to supply or remove hydrophobic substrates and products. This study investigates co-solvents of different polarities and structures as secondary solvents for the whole-cell alkane biooxidation. We found the polarity of co-solvents determines the accumulation of either alcohol or acid products and by choosing a more polar co-solvent the alcohol product could be increased. This suggests that the application of co-solvents is a promising strategy to influence the product specificity of whole cell bioconversions.

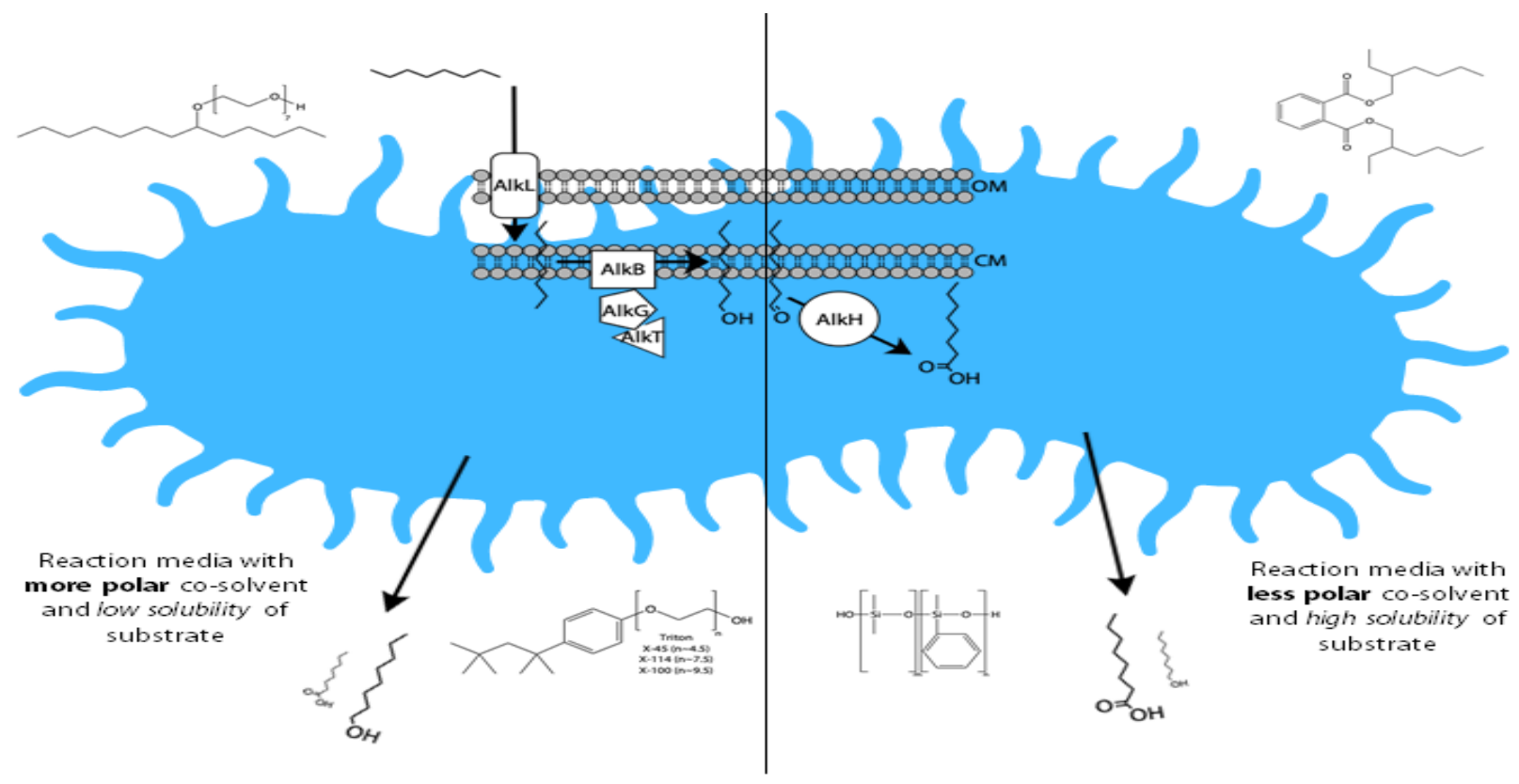

This article is protected by copyright. All rights reserved. 


\section{Introduction}

The application of two-liquid phase media has long been established to supply hydrophobic organic [1-6] and/or remove organic reaction products in situ [7-10] to reduce their inhibitory and toxic effects on the biocatalyst. In case of the whole-cell $\omega$ oxyfunctionalisation of linear alkanes by the AlkBGT enzyme system, the excess alkane substrate is often used as a second phase for product extraction. Moreover, addition and selection of inert, non-toxic and non-aqueous secondary solvents or surfactants have been shown to impact yields [11], biocompatibility, downstream processing [12] and ultimately biocatalyst efficiency [13] and process economics [14] of the AlkBGT mediated oxidation. Thus, the screening and optimisation of non-conventional media is of major importance for process development and performance to fully leverage the biocatalytic capability of the whole-cell biocatalyst [10, 15].

However, this selection of carrier phases or surfactants is difficult due to the large amount of parameters that are involved, such as: biocompatibility, substrate bioavailability, product yield, environmental impact, partition coefficient and extraction selectivity. The last parameter is especially applicable for controlling the specificity of the AlkBGT mediated oxidation and its product spectrum of primary alcohol, aldehyde and acid, where over-oxidation often leads to accumulation of the acid product. Overall, there is no clear guide for solvent selection making an empirical approach necessary. In addition to the aqueous phase and organic substrate phase, a third reaction medium component is referred to as a co-solvent in this article.

There have been approaches to identify co-solvents rationally and design nonconventional media for biological reactions based on basic physical parameters of substrate and co-solvents. Brink and Tramper [2] related the Hildebrand solubility parameter $(\delta)$ and molecular size measurements of various organic solvents to biocatalytic activity of whole-cells. However, the Hildebrand parameter is only applicable to largely non-polar, non-hydrogen bonding solvents. Similarly, the alternative hydrophilic-lipophilic balance (HLB) system is only applicable to classify nonionic surfactants according to their water or oil solubility and is consistent only within homologous series of such surfactants [16].

Most prominently, Laane et al. [17] simplified the approach of Brink and Tramper [2] by using the logarithm of the partition coefficient in a biphasic 1-octanol and water system for a particular solute $(\log P)$ as a superior descriptor of the solute's polarity and biocompatibility. The authors proposed the general rule that solvents with a $\log P>4$ are suitable for biocatalytic applications.

This article is protected by copyright. All rights reserved. 
Further, Laane et al. [17] suggests that by matching the polarity $(\log P)$ of the biocatalystcontinuous interphase to that of the substrate and/or using a continuous organic phase with different polarity than the substrate, the substrate concentration in the interphase can be maximised resulting in optimum activity of the biocatalyst in a micelle. Similarly, the polarity of the organic phase can be matched to the product polarity to extract the product away from the biocatalyst to avoid product inhibition or toxicity and shift the reaction equilibrium into the desired direction. Lastly, optimisation of the polarity of substrate and interphase can be important when substrate inhibition occurs.

Use of the $\log P$ parameter is useful as it can be predicted based on the molecular structure of the solvent independent of experimental measurements. Nevertheless, the impact of other factors such as the cell membrane and cell wall structure of a particular microorganism make empirical measurements necessary to confirm the biocompatibility. For example, non-polar solvents such as alkanes are toxic mainly due to their accumulation in and disruption of cell membranes [18, 19]. Compounds with additional functional groups, such as aldehydes or epoxides, are usually more toxic than their $\log P$ value indicates due to their additional, specific chemical toxicity [20]. Consequently, Filho et al. [21], postulate that $\log P$ is a poor generalisation of solvent compatibility for biocatalytic reactions.

Schneider [22] suggested widening the scope of parameters that are used for correlation and prediction of biocatalytic activity in organic solvents. Instead of the one-dimensional $\log P$ or Hildebrand parameter, use of the three-dimensional Hansen solubility parameters [23] was proposed. The Hansen solubility space is composed of three independent parameters that describe the diversity of solute-solvent interactions namely, dispersive forces $\left(\delta_{d}\right)$, polar interactions $\left(\delta_{p}\right)$ and hydrogen bonding $\left(\delta_{h}\right)$. The vector sum of all three parameters gives the previously mentioned Hildebrand parameter $(\delta$ ) (Equation 1$)$.

$$
\delta=\left(\delta_{d}^{2}+\delta_{p}^{2}+\delta_{h}^{2}\right)^{0.5}
$$

The solubility of a compound is displayed in three-dimensional space with a geometric centre point $\left(\delta_{d}, \delta_{p}, \delta_{h}\right)$. In general, the solubility of two compounds in each other decreases with increasing distance from each other.

Since then, there have been efforts to gain more mechanistic and thermodynamic insight into specific biocatalytic reaction systems in multiphase non-conventional media to improve systematic predictability of these reactions [24]. For example, computer aided solvent selection [15, 25, 26] and integrated process and solvent design approaches [2729] have been developed. Moreover, in recent years, increased attempts have been made

This article is protected by copyright. All rights reserved. 
to move away from a heuristic selection approach and instead to rationally select optimal solvents a priori for either substrate supply or product removal in biocatalytic systems. Zhou et al. [30] used theoretical quantum-chemical descriptors to quantify solvent effects in chemical reactions. Similarly, work has increasingly focused on investigating the influence of non-aqueous solvent properties on bio-catalysts and -reactions [31] to improve understanding of underlying mechanistics. For example, the selection of polymers for two-phase partitioning bioreactors using thermodynamic models has been successfully shown $[32,33]$. However, due to the high complexity of biochemical reactions, the application of universal models to predict outcomes a priori remains difficult.

Advances in automation, online-analysis and the application of statistical methods have resulted in a range of high-throughput, scale-down tools that have been successfully applied to collect large amounts of reliable quantitative data for characterisation and optimisation of microbial processes [34-36].

However, challenges remain for a universal application of these tools [37]. This is particularly relevant for non-conventional media. So far, the focus has been on developing microscale tools for aqueous single-phase systems. The unique challenges associated with more complex non-conventional media result in only few examples of suitable specialised tools [38-41].

With modern high-throughput methods large amounts of reliable quantitative data can be gathered for a large variety of parameters. However, this in turn requires the use of appropriate data analytical tools for reliably extracting a maximum of valuable information. Besides the frequently used Design of Experiment (DoE), multivariate data analysis (MVDA) is an alternative method to systematically analyse complex data. Often these large datasets contain a battery of similar, correlated measurements that all describe this complex problem. In these cases, the analysis and modelling of one variable at a time is time consuming and does not capture the underlying behaviour of the system.

More advanced MVDA methods such as partial least squares projections to latent structures (PLS) allow modelling the fundamental association between matrices of Xvariables (factors) and Y-variables (responses) by a linear multivariate model. Unlike multiple linear regression, PLS can analyse large amounts of noisy, collinear and even incomplete variables in both X and Y [42]. To that end, PLS iteratively fits a few latent variables to a projection of the $\mathrm{X}$ dataset that describe the maximum variance in the $\mathrm{Y}$ space [43]. Ultimately, this results in a model with fewer dimensions than multiple linear regressions of individual responses making it easier to interpret especially when

This article is protected by copyright. All rights reserved. 
responses are correlated. These properties have led to an increased use of MVDA techniques for a wide range of applications in biotechnological research and development [44].

This study investigates co-solvents of different polarities and structures as secondary solvents for the whole-cell alkane bio-oxidation by AlkBGT. Initially, the impact of six co-solvents at two concentrations is investigated. Particular focus is on the overall product yield (refers to sum of all products formed from the alkane oxidation in a specific reaction) and specificity (refers to the ratio of alcohol to acid product formed in a specific reaction) of the bio-oxidation of four linear alkane substrates. In order to efficiently screen this wide range of experimental conditions, a high-throughput microwell platform specifically customised for non-conventional media is used [41]. In a second step experimental data is combined with estimated physicochemical properties of the co-solvents in a multivariate PLS regression analysis. This allows the identification of key properties of co-solvents that specifically affect the AlkBGT reaction in terms of product specificity and yields.

\section{Materials and methods}

\subsection{Strains and plasmids}

For all bioconversions Escherichia coli GEC137 pGEc47 $\Delta \mathrm{J}$ was used [45, 46]. pGEc47 $\Delta \mathrm{J}$ contains all alkane oxidation genes of the OCT plasmid of Pseudomonas putida GPo1, except the alcohol dehydrogenase gene alkJ.

\subsection{Media composition 2.2.1 Growth medium The aqueous phase used for} fermentations was as described by Kolmar et al. [41].

\subsubsection{Buffer composition}

For bioconversion reactions with resting cells a $150 \mathrm{mmol} \mathrm{l}^{-1}$ potassium phosphate buffer with thiamine, $5 \mathrm{mg} \mathrm{l}^{-1}$ ( $\mathrm{pH}$ 7.2) was supplemented with separately heat sterilised: $\mathrm{MgSO}_{4}\left(7 \mathrm{H}_{2} \mathrm{O}\right), 1 \mathrm{~g} \mathrm{l}^{-1}$; D-glucose, $5.5 \mathrm{~g} \mathrm{l}^{-1}$ (all Sigma-Aldrich, UK); $\mathrm{CaCl}_{2}\left(2 \mathrm{H}_{2} \mathrm{O}\right)$, $0.04 \mathrm{~g} \mathrm{l}^{-1}$ (Alfa Aesar, UK); and $1 \mathrm{ml} \mathrm{l}^{-1}$ of the trace elements solution described in Kolmar et al. [33]. Further, filter sterilised tetracycline (Sigma-Aldrich, UK), $10 \mathrm{mg} \mathrm{l}^{-1}$ were added. The $\mathrm{pH}$ was routinely measured at 7.1 after addition.

This article is protected by copyright. All rights reserved. 


\subsection{Bioconversion procedure in microwell plates}

Bioconversions were carried out in sealed, polytetrafluoroethylene (PTFE) (Radleys, UK) 24 deep square well (DSW) microwell plates (MWP) using buffered resting cells according to Kolmar et al. [41].

\subsection{Solvent mixture preparation}

All co-solvents were purchased from commercial sources (Sigma-Aldrich, UK) at the highest available purity; silicone oil was purchased from Acros Organics.

In case of Triton surfactants, the surfactant was added to the aqueous buffer used for bioconversion reactions. All other co-solvent dilutions were prepared beforehand in the respective alkane substrate to either $5 \%$ or $30 \%(\mathrm{v} / \mathrm{v})$ concentration and stored in screw cap glass vials. Triton X-100 was prepared at $0.1 \%(\mathrm{v} / \mathrm{v})$ final concentration in the aqueous buffer. Triton $\mathrm{X}-45 / \mathrm{X}-115$ was prepared at $5 \%$ or $30 \%(\mathrm{v} / \mathrm{v})$ relative to the organic phase, despite being diluted in the aqueous phase. In all cases, except when using Triton X-100, the absolute aqueous and substrate volumes were kept constant and cosolvents were added in addition to the volumes used in the control reaction without cosolvent. For example, in case of an octane reaction with 30 \% Tergitol 15-S-7, $100 \mu$ of the prepared octane in Tergitol mixture (300 $\mathrm{ml} \mathrm{l}^{-1}$ ) were added to $280 \mu \mathrm{l}$ aqueous buffer. For the control reaction without co-solvent $70 \mu \mathrm{l}$ of alkane substrate were added to 280 $\mu \mathrm{l}$ aqueous buffer. Reactions were performed at conditions determined in Kolmar et al. [41], unless otherwise mentioned.

\subsection{Analytical procedures}

\subsubsection{Product recovery from solvent mixtures}

Extraction efficiencies of reaction products in four co-solvents reaction mixtures (ethyl oleate, silicone oil, Tergitol 15-S-7, poloxamer L-61) were tested at $30 \%$ in the octane substrate. These were determined by spiking reaction mixtures without cells with known amounts of oxidation products. This was carried out in screw cap glass vials. Vials were mixed on a rotator disk for at least $2 \mathrm{~h}$ at room temperature before extraction of the biphasic mixtures with cyclohexane for GC analysis. After extraction for GC analysis and concentration determination, the amount of recovered product against the spiked amount was calculated. Generally over $90 \%$ of product was recovered from the reaction mixtures. In case of Tergitol 15-S-7, extraction yield drops to $70 \%$ for the major products.

This article is protected by copyright. All rights reserved. 


\subsubsection{Gas chromatography}

Bioconversions were first stopped by acidifying with $50 \mu \mathrm{l}^{-1}$ of $10 \mathrm{~mol} \mathrm{l}^{-1} \mathrm{H}_{3} \mathrm{PO}_{4}$, subsequently $500 \mu \mathrm{l}$ cyclohexane with an internal standard were added. Depending on the alkane substrate chain length, the 1 -alcohol with $n+2$ chain length was used as an internal standard at $0.4 \mathrm{~g} \mathrm{l}^{-1}$. After incubating on a thermomixer for $10 \mathrm{~min}$ at $50{ }^{\circ} \mathrm{C}$ and $1000 \mathrm{rpm}$ the samples were centrifuged and the organic phase removed.

Organic samples were analysed by GC-FID (Thermo Fisher Scientific, UK) equipped with a Rxi-5Sil MS column (30 m $\times 0.53 \mathrm{~mm} \times 1.5 \mu \mathrm{m}$; Restek, USA) using helium as carrier gas (constant flow rate, $5 \mathrm{ml} \mathrm{min}^{-1}$ ). Hexane, heptane and octane bioconversions were analysed with an injector and detector temperature of $250{ }^{\circ} \mathrm{C}$ and $280{ }^{\circ} \mathrm{C}$ respectively. The temperature program was set at $80{ }^{\circ} \mathrm{C}$ for $2 \mathrm{~min}$, increased to $200{ }^{\circ} \mathrm{C}$ at $20^{\circ} \mathrm{C}$ min $^{-1}$ with a final hold of $3 \mathrm{~min}$. Dodecane bioconversions were analysed with an injector and detector temperature of $270{ }^{\circ} \mathrm{C}$ and $280{ }^{\circ} \mathrm{C}$ respectively. A temperature program of $100{ }^{\circ} \mathrm{C}$ for $1 \mathrm{~min}$ with an increase to $270{ }^{\circ} \mathrm{C}$ at $20^{\circ} \mathrm{C} \mathrm{min}{ }^{-1}$ and a final hold of 1 min was used. Quantification was achieved using external standards. All reported concentrations are in relation to the aqueous reaction volume.

\subsection{Data analysis}

\subsubsection{Physicochemical property estimation}

Hansen and $\log P$ parameters were estimated for co-solvents and reaction substrate and products using COMSOquick (v. 1.4, COSMOlogic, Germany). The quantitative structure property relationships models of the software were used to predict both parameters [47]. The Hansen parameter for silicone oil was estimated from Adamska et al. [48] and Woiton [49].

The distance between two compounds in the Hansen space $\left(R_{S}\right)$ and hence their solubility in each other was estimated using Equation 2 according to Hansen [19]. Here, the inverse was used to allow easier interpretation in subsequent analyses, thus, higher values represent better solubility of the two compounds in each other.

$$
R_{S}=\frac{1}{4\left(\delta_{d 1}-\delta_{d 2}\right)^{2}+\left(\delta_{p 1}-\delta_{p 2}\right)^{2}+\left(\delta_{h 1}-\delta_{h 2}\right)^{2}}
$$

\subsubsection{Multivariate data analysis}

Partial least squares projections to latent structures (PLS) regression analysis was performed (SIMCA 13.0.3, Umetrics, Umeå, Sweden) for analysing multiple variables in

This article is protected by copyright. All rights reserved. 
one model [43]. Two matrices were constructed; the X-matrix contained the chemometric descriptors and experimental conditions, the Y-matrix contained experimental results from the screening experiment including the product concentrations and alcohol to acid ratios (see Supporting Information, Tables S1-3). The data were pre-processed to mean 0 and variance 1. PLS models were fitted with default settings using autofit for model cross-validation. Correlations between factors (Co-solvent, co-solvent concentration, $R_{S}$ values, $\log P$ values) and responses (product concentrations, alcohol to acid ratios) were assessed in loading plots. For interpretation a line was drawn from a selected variable through the origin and $\mathrm{X}$ - and $\mathrm{Y}$-variables were projected on the line. A positive correlation was determined for variables adjacent to each other; for variables opposite to each other a negative correlation was determined. Model quality was assessed by goodness of fit $\left(\mathrm{R}^{2}\right)$ and goodness of prediction $\left(\mathrm{Q}^{2}\right)$. Model validation was successfully carried out using permutation tests over 40 iterations.

\section{Results 3.1 Co-solvent selection}

Six co-solvents were selected from literature and based on commercial availability. These span a wide range of polarities and structures, from very apolar small molecules such as bis(2-ethylhexyl) phthalate (BEHP) to more polar, polymeric molecules such as the nonionic triblock copolymer poloxamer L-61 (Table 1). It is hypothesised that these co-solvents cause a variety of effects. The more polar compounds (Tergitol 15-S-17, L61 and Triton) would improve product extraction away from the biocatalyst and thereby alleviate product toxicity for improved overall yields. At the same time, the surfactants are likely to improve substrate access by promoting emulsion formation. This would result in increased yields, due to higher alkane substrate availability. In addition, reducing substrate limitations may favour the production of alcohol to over-oxidation. However, increased substrate availability may also result in higher toxicity leading to reduced cell viability and biocatalyst performance [50]. In addition, the surfactants have been shown to form cloud-point systems, which can facilitate product separation and phase recycling, potentially alleviating the additional cost compared to an alkane-only organic phase [51]. On the other hand, by use of the more apolar co-solvents (BEHP, silicone oil and ethyl oleate), it is expected that a dilution of the organic phase may reduce toxic hydrophobic compound concentrations and thereby mitigate their toxicity. Although BEHP has been widely used in the literature, ethyl oleate is included here as an environmentally friendly alternative [52]. Silicone oil is included as a chemically inert alternative [53].

This article is protected by copyright. All rights reserved. 
The range of different molecules exemplifies the complexity of co-solvent selection. In summary, for the alkane oxidation, the co-solvent approach attempts to potentially solve several problems: shielding the biocatalyst from excessive substrate or product toxicity whilst allowing substrate supply and selective extraction of products from the biocatalyst.

\subsection{Co-solvent screening}

In addition to the six co-solvents, control reactions were run without additional cosolvent and with $0.1 \%$ Triton X-100, as in previous research [41, 54]. The screening was carried out with $5 \%$ and $30 \%$ co-solvent in four different alkane substrates over $24 \mathrm{~h}$ giving a total of 56 reactions (Figure 1).

Large, systematic variations in product concentrations were recorded for the different reactions. A tendency towards over-oxidation to the acid product can be seen from the product concentrations, especially at the control conditions with no co-solvent. The aldehyde was found in trace amounts at its highest in all cases, suggesting that the alcohol oxidation is a rate-limiting step.

In case of the dodecane substrate (Figure 1D), high acid product concentrations are achieved only at low concentrations of Triton surfactants. Grant et al. [54] have previously found that Triton X-100 addition is required for emulsion formation and successful bioconversion of dodecane in microwells. The lower performance of the dodecane bioconversion with Tergitol and L-61 is likely due to differences in emulsion formation. In contrast to reactions of the shorter chain alkane substrate, the addition of co-solvents never results in a surplus of 1-dodecanol. Instead, over-oxidation to the dodecanoic acid occurs with seemingly no back-extraction of the alcohol product into the organic phase.

With regard to the remaining data, the highest average overall yields are achieved in reactions with the natural octane substrate $\left(18.8 \mathrm{mmol} \mathrm{l}^{-1}\right)$, decreasing with substrate carbon chain length (Figure 1C). Similarly, Beilen et al. [55] found that shorter n-alkane substrates show reduced conversion rates, with longer chain substrates such as dodecane showing no conversion for a whole-cell system. This suggests that the biocatalyst is ideally suited for substrates of around eight carbon in chain length. However, Beilen et al. [55] present in vitro results of a reconstituted AlkBGT system that shows conversion of longer chain substrates such as dodecane with similarly high rates to that of octane. This indicates that cellular uptake of longer chain alkanes is a key factor and outer membrane transporters such as AlkL are necessary for oxidation of longer-chain substrates [56].

This article is protected by copyright. All rights reserved. 
Further, the average molar alcohol to acid product ratio is highest for the octane reactions (2.1:1), decreasing with the other substrates. Therefore, similarly to the variation of total yields with chain length, the product spectrum is influenced by chain length.

In terms of the product spectrum, it is interesting to note that reactions with the Tergitol, L-61 and Triton co-solvents achieve very high molar alcohol to acid ratios, on average over all substrates the ratios were 3.5:1, 1.6:1 and 2.4:1 respectively. For hexane and heptane over seven fold higher alcohol than acid product formation was seen after $24 \mathrm{~h}$ in the presence of $30 \%$ Tergitol. Most interestingly, for the octane oxidation with $30 \%$ Tergitol, an alcohol to acid ratio of 5.7:1 was seen with $18.3 \mathrm{mmol}^{-1}$ final 1-octanol concentration. This represents a 3.2 fold increase in alcohol yield relative to the control reaction without co-solvents for which the acid product dominates with an alcohol to acid ratio of 0.4:1 whilst maintaining similar overall product yields.

In case of hexane, heptane and octane, the overall highest product quantities were achieved with the more nonpolar solvents, namely BEHP, silicone oil and also ethyl oleate. Up to $35.6 \mathrm{mmol} \mathrm{l}^{-1}$ of products were formed in case of the octane oxidation with $30 \%$ BEHP present. This represents a 1.7 times increase relative to the control reaction without co-solvent and 1.6 times higher than in the reaction with $30 \%$ Tergitol. The increase in overall yield with higher concentrations of these co-solvents is contrary to previous findings were BEHP was seen to limit substrate access and promote overoxidation $[57,58]$. However, in those studies, much lower substrate concentrations in BEHP were used compared to this study.3.2.1 Time course of bioconversion with selected co-solvents

In order to verify the co-solvent effects seen in the initial screening four co-solvents were chosen (ethyl oleate, silicone oil, L-61 and Tergitol at $30 \%$ in alkane substrate) and time course data was collected. The experiments were performed using the octane substrate and compared to a control reaction without co-solvent (Figure 2).

Overall, the trends of the screening were confirmed by the time course data. High average overall productivity was recorded over the initial $9 \mathrm{~h}$ in the presence of ethyl oleate (3.6 mmol $\left.\mathrm{l}^{-1} \mathrm{~h}^{-1}\right)$ and silicone oil $\left(2.8 \mathrm{mmol} \mathrm{l}^{-1} \mathrm{~h}^{-1}\right)$ with the octanoic acid product dominating. However, the increased productivity is at the detriment of product specificity with both, acid and 1-alcohol products being produced. This is in contrast to reactions with Tergitol and L-61 where higher specificity for the 1-octanol product with alcohol excess of 7.7:1 and 5.8:1 were recorded after $24 \mathrm{~h}$.

This article is protected by copyright. All rights reserved. 


\subsection{Multivariate data analysis of co-solvent effect on alkane bio- oxidations}

In order to gain further insight into the impact of physicochemical parameters of the cosolvents on the reaction yields, product specificity and whether an underlying relationship between these parameters and the reaction outcomes can be found, a PLS regression analysis approach was adopted. A particular focus was on investigating the ratio of alcohol to acid product. To avoid introducing severe non-linearity in the model the dodecane substrate was not considered in this analysis due to either very low conversion or large excess in dodecanoic acid.

\subsubsection{Physicochemical property estimation}

Two physicochemical parameters were chosen to describe all reaction components, the substrates and products as well as the co-solvent. The $\log P$ values were used to investigate the influence on the reaction according to Laane et al. [17]. The Hansen solubility parameters were used to derive solubility properties $\left(R_{S}\right)$ of reaction substrates and products in co-solvents to be used as factors together with the substrate and cosolvent identity, co-solvent concentration and substrate carbon chain length. The molar reaction product concentrations as well as the ratio of alcohol to acid after $24 \mathrm{~h}$ of the initial screening experiments were taken as responses.

\subsubsection{Model summary}

An initial PLS model (model M1) was created from the data resulting in three components showing good correlation between the two matrices. The loading plot provides an overview of the interactions between factors and responses of the system displaying the first two components (see SI, Figure S1). Here, the $\log P$ parameters for the substrate and products correlated strongly with the substrate carbon chain length and unsurprisingly with the $R_{S}$ value for substrate solubility in water, however, only showing significance in the second component. The linear nature of the alkane substrate and hence products simplify the correlation of these parameters. Thus, $\log P$ of the substrate can be used exemplarily for all compounds in the dataset. The $\log P$ of co-solvents shows less influence on the model being close to the origin. Based on this initial appraisal the $\log P$ values for co-solvent and products, $R_{S}$ Subs-Water parameter and the substrate chain length were removed from the initial model to simplify the model.

The new PLS model (model M2) found two significant components, with overall good values for goodness of fit $\left(\mathrm{R}^{2} \mathrm{Y}\right)$ and prediction $\left(\mathrm{Q}^{2} \mathrm{Y}\right)$ (Table 2). The lower explained 
variation values for the factors $\left(\mathrm{R}^{2} \mathrm{X}\right)$ of the model can be caused by the large number of categorical variables in the $\mathrm{X}$ data. Overall, with good fit and predictive power, the model shows a strong, quantitative relationship between the factors and responses.

\subsubsection{Model evaluation}

From the score plot of the PLS model (Figure 3A) it can be seen that the response data is split by co-solvent type in two groups. The data in the Y-space clearly group according to the used co-solvent; the apolar co-solvents (BEHP, silicone oil, ethyl oleate) on the left and the more polar co-solvents on the right (Tergitol, Triton, L-61). The control samples without co-solvent are associated with the left group.

When consulting the corresponding loading plot of the PLS model, it is evident that the first component along the x-axis includes the polarity properties of the co-solvent,here specifically represented as the solubility $R_{S}$ values of co-solvent in substrate or alcohol and acid products (Figure 3B). From the clustering in Figure 3B it can be seen that substrates show better solubility in apolar solvents and the alcohol and acid products show better solubility in polar solvents. Interestingly, there is an association of high alcohol to acid ratio with good solubility of products in the co-solvent, especially Tergitol.

The second component along the $y$-axis is mostly described by the $\log P$ of the substrate and the co-solvent concentration (Figure $3 \mathrm{~B}$ ), bearing in mind that the $\log P$ for substrates is correlated with the $\log P$ for alcohol and acid products (see SI, Figure S1).

The interactions between both components summarise the effects over the entire dataset. Co-solvents such as BEHP or ethyl oleate result in high total product yields mainly due to high acid yields. On the other side, Tergitol results in high alcohol over acid ratio, but not in high total yields, there is a weak association with high alcohol yields similar to BEHP, though. In contrast, the Triton co-solvents have an overall negative effect on the reaction. Silicone oil has little effect on the reaction relative to the control reaction without co-solvent. The slight association of acid yield with total product yield shows that the former is the preferred product and high alcohol over acid ratios being opposed to this.

Further, higher $\log P$ values and higher co-solvent concentration specifically result in higher alcohol yield. This is a good indication of organic compound toxicity as proposed by Laane et al. [17], with toxicity decreasing with an increase in $\log P(\log P>4)$. The association of this parameter with the alcohol yield suggests that toxicity is specifically associated with the alcohol product. The high toxicity of the alcohol product explains the

This article is protected by copyright. All rights reserved. 
difficulty of achieving high alcohol yields in these reactions. Further, it illustrates the need for in situ product removal to reduce toxic effects towards the whole-cell biocatalyst and the resulting reduction in productivity. The positive correlation with the co-solvent concentration confirms their positive impact specifically on the alcohol yield. It can be speculated that this is in fact due to the extraction of the alcohol into the cosolvent due to preferential solubilisation in the co-solvent. This can also be seen by the association of the $R_{S}$ value for co-solvent in alcohol with Tergitol. In addition, this effect can simply be due to the larger volume of co-solvent available for solubilisation. On the other hand, high acid yields are achieved when maximising the solubility of substrates in co-solvents and essentially diluting the substrate. This reduces substrate access and promotes over-oxidation as previously argued [58].

The Variable Influence on Projection (VIP) plot of the PLS model (see SI, Figure S2) is a ranking of the influence of each factor on the response variables; values larger than 1 are generally considered highly important, and values larger than 0.5 are considered relevant [59]. Due to the large amount of categoric data and the associated variation, the cosolvent variables have a low score with large estimated confidence interval.

Nevertheless, these variables remain central to the model. Overall, the most influential and reliable factor is the $R_{S}$ co-solvent in substrate value $(\mathrm{VIP}=1.4)$ followed by the substrate $\log P(\mathrm{VIP}=1.3)$.

This reiterates the importance of the $R_{S}$ values for solubility of either substrate or products in co-solvent and the substrate $\log P$. Whilst this shows the general influence of substrate $\log P$ values on the bio-oxidation of alkanes, it provides a simplistic view and the impact of individual products cannot be captured with this highly correlated variable. It is interesting to see the strong correlation of the $\log P$ for substrate specifically with the alcohol product (see Figure 3B), suggesting that the alcohol product is primarily responsible for toxicity. Despite its own limitations, the application of $R_{S}$ values has been shown to be more insightful than $\log P$ values, especially for this complex multi-product reaction.

Finally, it is interesting to note that there is a clear separation of co-solvents by their ability to solubilise the reaction products. The solubility of substrate relative to products in the co-solvents indicates that preferential solubilisation of the product leads to an extraction away from the biocatalyst resulting in higher alcohol over acid ratios. For high absolute alcohol yields, the alcohol toxicity needs to be successfully mitigated, as well. On the other hand, with high substrate solubility in the co-solvent the substrate gets diluted and retained in the organic phase, resulting in low mass transfer of substrate to the biocatalyst promoting the over-oxidation of 1-alcohol to acid [58].

This article is protected by copyright. All rights reserved. 


\section{Discussion}

The presented data shows the large impact that non-conventional media composition has on the whole-cell bio-oxidation of alkanes by the AlkBGT enzyme system. By successfully leveraging a high-throughput scale-down tool in combination with chemometric multivariate data analysis we rapidly gained an understanding of the underlying factors that relate media composition to product yields and specificity. Especially with $30 \%$ Tergitol as co-solvent and the octane substrate, a 3.2 fold improvement in alcohol yield relative to the control reaction without co-solvents was achieved. At the same time the alcohol to acid ratio increased from $0.4: 1$ to $5.6: 1$. The fact that this was achieved with the unoptimised screening conditions shows the potential of this approach for controlling the reaction product spectrum.

The highest overall yields were achieved using BEHP and ethyl oleate. In case of reactions with $30 \% \mathrm{BEHP}$, up to $36 \mathrm{mmol} \mathrm{l}^{-1}$ of products were formed with the octane substrate, representing a 1.7 fold improvement relative to the control reaction without cosolvent and 1.6 times higher than the reaction with 30 \% Tergitol.Generally, the use of more polar co-solvents such as Tergitol markedly reduced the over-oxidation of octane reaction products and an accumulation of 1-octanol was observed. In contrast, the apolar ethyl oleate showed improved overall yields with the acid product dominating.

PLS regression of the co-solvent screening dataset showed that the defining factor for product specificity are the solubility properties of the co-solvent. The polar co-solvents are generally better solvents for the reaction products. This allows the conclusion that in this case, the products are in fact extracted away from the biocatalyst, resulting in reduced toxicity especially due to the alcohol product.

The Hansen solubility framework that was used in the analysis can provide a universal and accessible overview of the impact of co-solvents on the reaction yields of whole-cell alkane oxidations. Especially the solubility parameters $\left(R_{S}\right)$ for substrate and product in the co-solvent have been shown to be highly significant for product yields and specificity. This makes use of the $R_{S}$ parameter preferable to the one-dimensional $\log P$.

Large margins for optimisation based on the presented screening data remain. The choice of co-solvent can strongly influence the reaction outcomes. It is likely that local optima exist that have not been captured or characterised in the present study. For example, some of the used co-solvents are commercially available with a large range of permutations, such as chain length of poloxamers. The $R_{S}$ value of these potential cosolvents can be used to select suitable candidates for evaluation. Further, the phase ratio

This article is protected by copyright. All rights reserved. 
of co-solvent to substrate and aqueous phase presents an optimisation target. For example, it is likely that there is an optimum BEHP concentration that allows maximum overall product yields, before either limiting substrate access (at higher BEHP concentrations) or ineffectively mitigating substrate and product toxicity (at lower BEHP concentrations).

This study shows the importance of two-liquid phase reaction engineering for optimising and directing biocatalytic reactions. The combination with computational and statistical tools was shown to be highly effective at pooling a wide range of experimental data, simplifying its interpretation and maximising its information output. The microwell platform allows integration of biocatalyst, process and reaction engineering early in development. The high fidelity data gained can help explore various options rapidly with high confidence.

However, the use of co-solvents and respective improvements in product yield and specificity need to weighed against complications in downstream processing. For example, the formation of stable emulsions can make separation of the organic phase and product recovery difficult [60-63]. The ease of phase separation and product recovery has a direct impact on overall economics of the alkane oxidation process [64]. Thus, it is crucial to consider the downstream processing options to completely evaluate the potential use of co-solvents for alkane bio-oxidations.

Overall, the application of co-solvents is a promising strategy to influence whole-cell alkane oxidations. Further work needs to investigate efficient downstream processing options for the most promising candidates to fully leverage their advantages.

\section{Acknowledgments}

Funding from EPSRC, Industrial Doctorate Centre (EP/G034656/1).

\section{Conflict-of-Interest statement}

The authors declare no commercial or financial conflict of interest.

\section{References}

[1] T Nakahara, LE Erickson, JR Gutierrez, Characteristics of hydrocarbon uptake in cultures with two liquid phases. Biotechnol. Bioeng. 1977, 19, 9-25.

[2] LE Brink, J Tramper, Optimization of organic solvent in multiphase biocatalysis. Biotechnol. Bioeng. 1985, 27, 1258-1269.

This article is protected by copyright. All rights reserved. 
[3] MG Wubbolts, J Hoven, B Melgert, B Witholt, Efficient production of optically active styrene epoxides in two-liquid phase cultures. Enzyme Microb. Technol. 1994, 16, 887-894.

[4] E Déziel, Y Comeau, R Villemur, Two-liquid-phase bioreactors for enhanced degradation of hydrophobic/toxic compounds. Biodegradation 1999, 10, 219-233.

[5] P-Y Kim, DJ Pollard, JM Woodley, Substrate supply for effective biocatalysis. Biotechnol. Prog. 2007, 23, 74-82.

[6] G Darracq, A Couvert, C Couriol, A Amrane, P Le Cloirec, Removal of Hydrophobic Volatile Organic Compounds in an Integrated Process Coupling Absorption and Biodegradation-Selection of an Organic Liquid Phase. Water, Air, \&amp; Soil Pollution 2012, 223, 4969-4997.

[7] AJ Daugulis, Integrated Reaction and Product Recovery in Bioreactor Systems. Biotechnol. Prog. 1988, 4, 113-122.

[8] JM Woodley, M Bisschops, AJJ Straathof, M Ottens, Future directions for in-situ product removal (ISPR). Journal of Chemical Technology \&amp; Biotechnology 2008, 83, 121-123.

[9] Z Wang, Z Dai, Extractive microbial fermentation in cloud point system. Enzyme Microb. Technol. 2010, 46, 407-418.

[10] JT Dafoe, AJ Daugulis, In situ product removal in fermentation systems: improved process performance and rational extractant selection. Biotechnol. Lett. 2013, 36, 443460.

[11] C Grant, JM Woodley, F Baganz, Whole-cell bio-oxidation of n-dodecane using the alkane hydroxylase system of P. putida GPo1 expressed in E. coli. Enzyme Microb. Technol. 2011, 48, 480-486.

[12] RG Mathys, OM Kut, B Witholt, Alkanol removal from the apolar phase of a twoliquid phase bioconversion system. Part 1: Comparison of a less volatile and a more volatile in-situ extraction solvent for the separation of 1-octanol by distillation. Journal of Chemical Technology \&amp; Biotechnology 1998, 71, 315-325.

[13] D Kuhn, FSO Fritzsch, X Zhang, VF Wendisch, LM Blank, B Bühler, A Schmid, Subtoxic product levels limit the epoxidation capacity of recombinant E. coli by increasing microbial energy demands. J. Biotechnol. 2013, 163, 194-203.

[14] A Schmid, B Sonnleitner, B Witholt, Medium chain length alkane solvent ～- cell transfer rates in two-liquid phase, Pseudomonas oleovorans cultures. Biotechnol. Bioeng. 1998, 60, 10-23.

[15] LJ Bruce, AJ Daugulis, Solvent selection strategies for extractive biocatalysis. Biotechnol. Prog. 1991, 7, 116-124.

This article is protected by copyright. All rights reserved. 
[16] H Schott, Hydrophilic-Lipophilic Balance, Solubility Parameter, and Oil-Water Partition Coefficient as Universal Parameters of Nonionic Surfactants. J. Pharm. Sci. 1995, 84, 1215-1222.

[17] C Laane, S Boeren, K Vos, C Veeger, Rules for optimization of biocatalysis in organic solvents. Biotechnol. Bioeng. 1987, 30, 81-87.

[18] J Sikkema, JAM de Bont, B Poolman, Mechanisms of membrane toxicity of hydrocarbons. Microbiol. Mol. Biol. Rev. 1995, 59, 201-222.

[19] HJ Heipieper, PM Martínez, Toxicity of Hydrocarbons to Microorganisms, Springer Berlin Heidelberg, Berlin, Heidelberg 2010, pp. 1563-1573.

[20] MH Vermuë, J Sikkema, A Verheul, R Bakker, J Tramper, Toxicity of homologous series of organic solvents for the gram-positive bacteria Arthrobacter and Nocardia Sp. and the gram-negative bacteria Acinetobacter and Pseudomonas Sp. Biotechnol. Bioeng. 1993, 42, 747-758.

[21] MV Filho, T Stillger, M Müller, A Liese, C Wandrey, Is logP a convenient criterion to guide the choice of solvents for biphasic enzymatic reactions? Angew. Chem. Int. Ed. 2003, 42, 2993-2996.

[22] LV Schneider, A three-dimensional solubility parameter approach to nonaqueous enzymology. Biotechnol. Bioeng. 1991, 37, 627-638.

[23] CM Hansen, Solubility Parameters, in: JV Koleske (Ed.), ASTM International, West Conshohocken, PA 2012, pp. 470-494.

[24] PJ Halling, Thermodynamic predictions for biocatalysis in nonconventional media: Theory, tests, and recommendations for experimental design and analysis. Enzyme Microb. Technol. 1994, 16, 178-206.

[25] MH Vermuë, J Tramper, Biocatalysis in non-conventional media: medium engineering aspects. Pure Appl. Chem. 1995, 67.

[26] J Abildskov, MB van Leeuwen, CG Boeriu, LAM van den Broek, Computer-aided solvent screening for biocatalysis. J. Mol. Catal. B: Enzym. 2013, 85-86, 200-213.

[27] Y Wang, LEK Achenie, Computer aided solvent design for extractive fermentation. Fluid Phase Equilib. 2002, 201, 1-18.

[28] HC Cheng, FS Wang, Computer-aided biocompatible solvent design for an integrated extractive fermentation-separation process. Chem. Eng. J. 2010, 162, 809-820.

[29] L Moity, V Molinier, A Benazzouz, B Joossen, V Gerbaud, J-M Aubry, A “topdown” in silico approach for designing ad hoc bio-based solvents: application to glycerol-derived solvents of nitrocellulose. Green Chemistry 2016, 18, 3239-3249.

[30] T Zhou, Z Qi, K Sundmacher, Model-based method for the screening of solvents for chemical reactions. Chem. Eng. Sci. 2014, 115, 177-185.

This article is protected by copyright. All rights reserved. 
[31] D Lousa, AM Baptista, CM Soares, A molecular perspective on nonaqueous biocatalysis: contributions from simulation studies. PCCP 2013, 15, 13723.

[32] SL Bacon, JS Parent, AJ Daugulis, A framework to predict and experimentally evaluate polymer-solute thermodynamic affinity for two-phase partitioning bioreactor (TPPB) applications. Journal of Chemical Technology \&amp; Biotechnology 2014, 89, 948-956.

[33] SL Bacon, EC Peterson, AJ Daugulis, JS Parent, Selecting polymers for two-phase partitioning bioreactors (TPPBs): Consideration of thermodynamic affinity, crystallinity, and glass transition temperature. Biotechnol. Prog. 2015, 31, 1500-1507.

[34] Q Long, X Liu, Y Yang, L Li, L Harvey, B McNeil, Z Bai, The development and application of high throughput cultivation technology in bioprocess development. $J$. Biotechnol. 2014, 192, 323-338.

[35] J Hemmerich, S Noack, W Wiechert, M Oldiges, Microbioreactor Systems for Accelerated Bioprocess Development. Biotechnol J 2018, 13, e1700141.

[36] ML Velez-Suberbie, JPJ Betts, KL Walker, C Robinson, B Zoro, E KeshavarzMoore, High throughput automated microbial bioreactor system used for clone selection and rapid scale-down process optimization. Biotechnol. Prog. 2018, 34, 58-68.

[37] T Tajsoleiman, L Mears, U Kruhne, KV Gernaey, S Cornelissen, An Industrial Perspective on Scale-Down Challenges Using Miniaturized Bioreactors. Trends Biotechnol. 2019, 10.1016/j.tibtech.2019.01.002.

[38] MPC Marques, F Carvalho, S Magalhães, JMS Cabral, P Fernandes, Screening for suitable solvents as substrate carriers for the microbial side-chain cleavage of sitosterol using microtitre plates. Process Biochem. 2009, 44, 556-561.

[39] H Giese, P Kruithof, K Meier, M Sieben, E Antonov, RWJ Hommes, J Büchs, Improvement and scale-down of a Trichoderma reesei shake flask protocol to microtiter plates enables high-throughput screening. J. Biosci. Bioeng. 2014, 118, 702-709.

[40] T Schlepütz, J Büchs, Scale-down of vinegar production into microtiter plates using a custom-made lid. J. Biosci. Bioeng. 2014, 117, 485-496.

[41] JF Kolmar, O Thum, F Baganz, Customized microscale approach for optimizing two-phase bio-oxidations of alkanes with high reproducibility. Microbial Cell Factories 2017, 16, 174.

[42] L Eriksson, T Byrne, E Johansson, J Trygg, C Vikström, Multi- and Megavariate Data Analysis, MKS Umetrics AB, Malmö, Sweden 2013.

[43] S Wold, M Sjöström, L Eriksson, PLS-regression: a basic tool of chemometrics. Chemometrics Intellig. Lab. Syst. 2001, 58, 109-130.

[44] AS Rathore, N Bhushan, S Hadpe, Chemometrics applications in biotech processes: a review. Biotechnol. Prog. 2011, 27, 307-315.

This article is protected by copyright. All rights reserved. 
[45] G Eggink, RG Lageveen, B Altenburg, B Witholt, Controlled and functional expression of the Pseudomonas oleovorans alkane utilizing system in Pseudomonas putida and Escherichia coli. J. Biol. Chem. 1987, 262, 17712-17718.

[46] JB van Beilen, G Eggink, H Enequist, R Bos, B Witholt, DNA sequence determination and functional characterization of the OCT-plasmid-encoded alkJKL genes of Pseudomonas oleovorans. Mol. Microbiol. 1992, 6, 3121-3136.

[47] C Loschen, A Klamt, COSMOquick: A Novel Interface for Fast $\sigma$-Profile Composition and Its Application to COSMO-RS Solvent Screening Using Multiple Reference Solvents. Industrial \&amp; Engineering Chemistry Research 2012, 51, 14303 14308.

[48] K Adamska, A Voelkel, K Héberger, Selection of solubility parameters for characterization of pharmaceutical excipients. J. Chromatogr. 2007, 1171, 90-97.

[49] M Woiton, Universität Erlangen-Nürnberg 2014.

[50] M Kadisch, M Julsing, M Schrewe, N Jehmlich, B Scheer, M von Bergen, A Schmid, B Bühler, Maximization of cell viability rather than biocatalyst activity improves whole-cell $\omega$-oxyfunctionalization performance. Biotechnol. Bioeng. 2017, 114, 874-884.

[51] Z Wang, The potential of cloud point system as a novel two-phase partitioning system for biotransformation. Appl. Microbiol. Biotechnol. 2007, 75, 1-10.

[52] D Kuhn, M Julsing, E Heinzle, B Bühler, Systematic optimization of a biocatalytic two-liquid phase oxyfunctionalization process guided by ecological and economic assessment. Green Chemistry 2012, 14, 645.

[53] S El Aalam, A Pauss, JM Lebeault, High efficiency styrene biodegradation in a biphasic organic/water continuous reactor. Appl. Microbiol. Biotechnol. 1993, 39, 696699.

[54] C Grant, AC da Silva Damas Pinto, H-P Lui, JM Woodley, F Baganz, Tools for characterizing the whole-cell bio-oxidation of alkanes at microscale. Biotechnol. Bioeng. 2012, 109, 2179-2189.

[55] JB van Beilen, J Kingma, B Witholt, Substrate specificity of the alkane hydroxylase system of Pseudomonas oleovorans GPo1. Enzyme Microb. Technol. 1994, 16, 904-911.

[56] C Grant, D Deszcz, Y-C Wei, RJ Martínez-Torres, P Morris, T Folliard, R Sreenivasan, J Ward, P Dalby, JM Woodley, F Baganz, Identification and use of an alkane transporter plug-in for applications in biocatalysis and whole-cell biosensing of alkanes. Scientific Reports 2014, 4.

[57] S Cornelissen, M Julsing, J Volmer, O Riechert, A Schmid, B Bühler, Whole-cellbased CYP153A6-catalyzed (S)-limonene hydroxylation efficiency depends on host background and profits from monoterpene uptake via AlkL. Biotechnol. Bioeng. 2013, $110,1282-1292$

This article is protected by copyright. All rights reserved. 
[58] M Schrewe, M Julsing, K Lange, E Czarnotta, A Schmid, B Bühler, Reaction and catalyst engineering to exploit kinetically controlled whole-cell multistep biocatalysis for terminal FAME oxyfunctionalization. Biotechnol. Bioeng. 2014, 111, 1820-1830.

[59] M Umetrics, User Guide to SIMCA 13, Malmö, Sweden 2012.

[60] A Schmid, A Kollmer, RG Mathys, B Witholt, Developments toward large-scale bacterial bioprocesses in the presence of bulk amounts of organic solvents. Extremophiles 1998, 2, 249-256.

[61] H Cheng, DA Sabatini, Separation of Organic Compounds from Surfactant Solutions: A Review. Sep. Sci. Technol. 2007, 42, 453-475.

[62] C Brandenbusch, S Glonke, J Collins, R Hoffrogge, K Grunwald, B Bühler, A Schmid, G Sadowski, Process boundaries of irreversible scCO 2-assisted phase separation in biphasic whole-cell biocatalysis. Biotechnol. Bioeng. 2015, 112, 23162323.

[63] J Collins, M Grund, C Brandenbusch, G Sadowski, A Schmid, B Bühler, The dynamic influence of cells on the formation of stable emulsions in organic-aqueous biotransformations. Journal of Industrial Microbiology \&amp; Biotechnology 2015, 42, 1011-1026.

[64] RG Mathys, A Schmid, OM Kut, B Witholt, Alkanol removal from the apolar phase of a two-liquid phase bioconversion system. Part 2: Effect of fermentation medium on batch distillation. Journal of Chemical Technology \&amp; Biotechnology 1998, 71, 326334.

[65] A Cruz, P Fernandes, JMS Cabral, HM Pinheiro, Solvent partitioning and wholecell sitosterol bioconversion activity in aqueous-organic two-phase systems. Enzyme Microb. Technol. 2004, 34, 342-353.

[66] F Carvalho, MPC Marques, CCCR de Carvalho, JMS Cabral, P Fernandes, Sitosterol bioconversion with resting cells in liquid polymer based systems. Bioresour. Technol. 2009, 100, 4050-4053.

[67] Z Wang, F Zhao, X Hao, D Chen, D Li, Microbial transformation of hydrophobic compound in cloud point system. J. Mol. Catal. B: Enzym. 2004, 27, 147-153.

[68] Z Wang, JH Xu, W Zhang, B Zhuang, H Qi, Cloud point of nonionic surfactant Triton X-45 in aqueous solution. Colloids Surf. B. Biointerfaces 2008, 61, 118-122.

[69] P Glembin, M Kerner, I Smirnova, Cloud point extraction of microalgae cultures. Sep. Purif. Technol. 2013, 103, 21-27.

[70] PB Dhamole, Z Wang, Y Liu, B Wang, H Feng, Extractive fermentation with nonionic surfactants to enhance butanol production. Biomass Bioenergy 2012, 40, 112-119.

This article is protected by copyright. All rights reserved. 


\section{Tables}

Table 1: Overview of tested co-solvents.

\begin{tabular}{llll}
\hline Co-solvent & Structure & Details & References \\
\hline $\begin{array}{l}\text { Bis(2-ethyl-hexyl) } \\
\text { phthalate }\end{array}$ & $\begin{array}{l}\text { Non-toxic organic carrier } \\
\text { phase for whole-cell FAME } \\
\text { oxidation; promotes over- } \\
\text { oxidation }\end{array}$ \\
Silicone oil & $\begin{array}{l}\text { Non-toxic organic carrier } \\
\text { phase for whole-cell } \\
\text { phytosterol cleavage }\end{array}$ & \\
\end{tabular}

Ethyl oleate

Non-toxic organic carrier

$[52,58]$

phase for whole-cell FAME

oxidation; promotes over-

oxidation

Triton X-Series

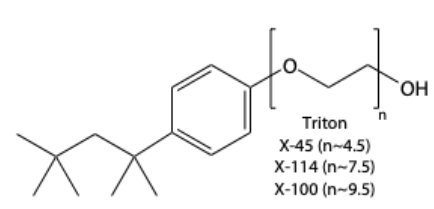

Biocompatible carrier phase

$[67,68]$

for whole-cell bioconversion

of sterols

Tergitol 15-S-7

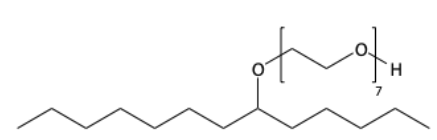

Biocompatible fatty acid

extraction from algal

cultures; recycling of

surfactant

Poloxamer L-61

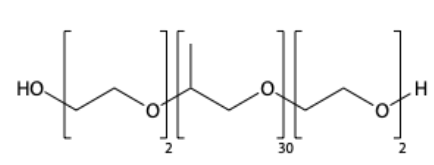

Selective butanol extraction

[70]

from $\mathrm{ABE}$ fermentation with

L-62 (2× EO ${ }^{b}$ of L-61);

recycling of surfactant

\footnotetext{
${ }^{a}$ Fatty acid methyl ester

${ }^{b}$ Ethylene oxide
}

This article is protected by copyright. All rights reserved. 
Table 2: PLS model M2 parameters, cumulative coefficients for goodness of fit $\left(\mathrm{R}^{2} \mathrm{Y}\right)$, goodness of prediction $\left(\mathrm{Q}^{2} \mathrm{Y}\right)$ and explained variation $\left(\mathrm{R}^{2} \mathrm{X}\right)$.

\begin{tabular}{llll}
\hline Component & $\mathrm{R}^{2} \mathrm{X}$ & $\mathrm{R}^{2} \mathrm{Y}$ & $\mathrm{Q}^{2} \mathrm{Y}$ \\
\hline & $1^{0.24}$ & 0.47 & 0.35 \\
& $2^{0.35}$ & 0.75 & 0.62 \\
\hline
\end{tabular}

\section{Figures}

Figure 1: Bioconversion of hexane (A), heptane (B), octane (C) and dodecane (D) substrate after $24 \mathrm{~h}$ at $30{ }^{\circ} \mathrm{C}$ and $250 \mathrm{rpm}$, with varying co-solvent percentages in substrate indicated below $\mathrm{X}$-axis, except TX-100 in aqueous buffer.
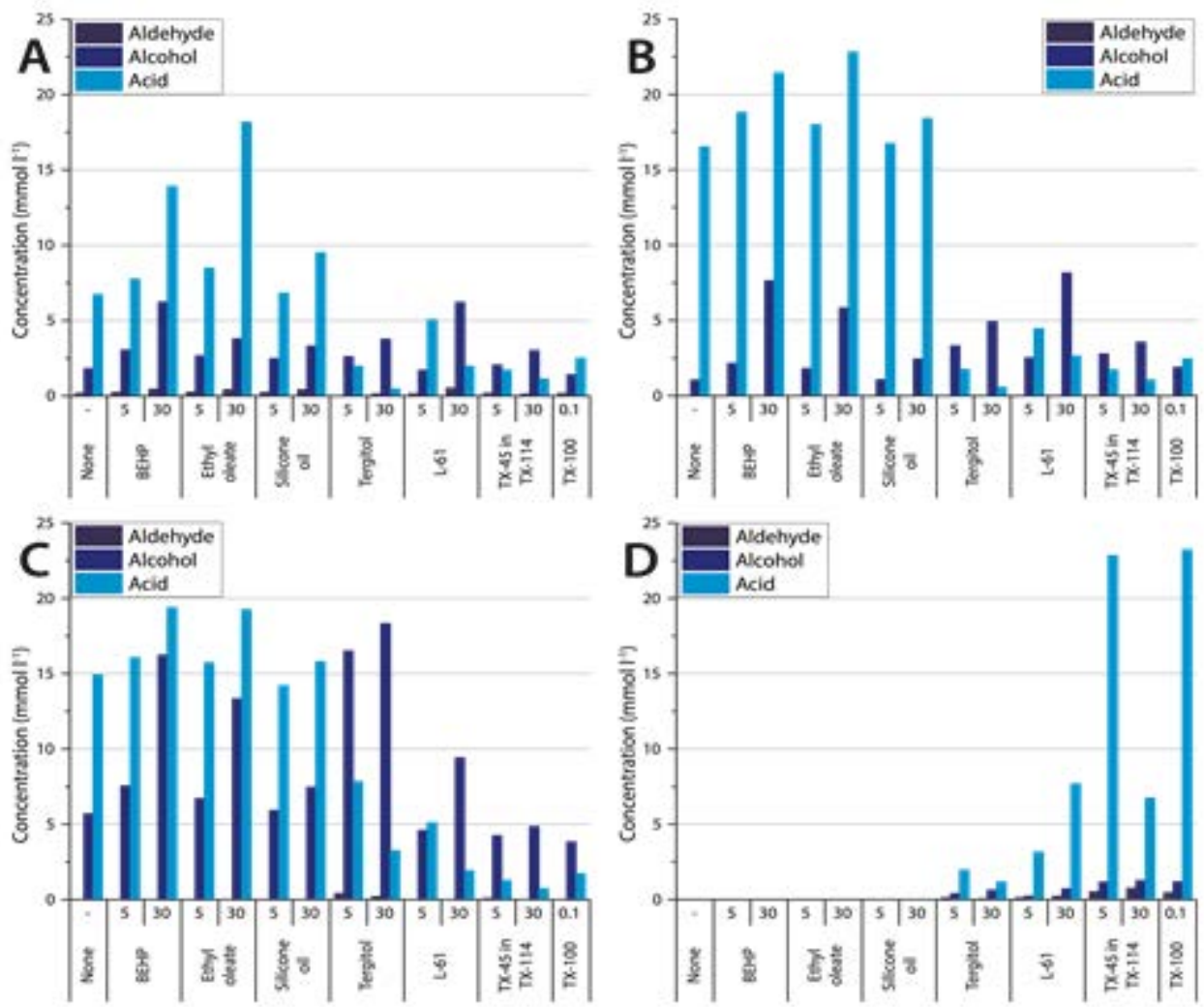

This article is protected by copyright. All rights reserved. 
Figure 2: Bioconversion time course with co-solvents: 30 \% ethyl oleate (A), silicone oil (B), Tergitol (C), L-61 (D) or no co-solvent (E) in octane over $24 \mathrm{~h}$ at $30{ }^{\circ} \mathrm{C}$ and 250 $\mathrm{rpm} ; \mathrm{n}=2, \pm \mathrm{SD}$.
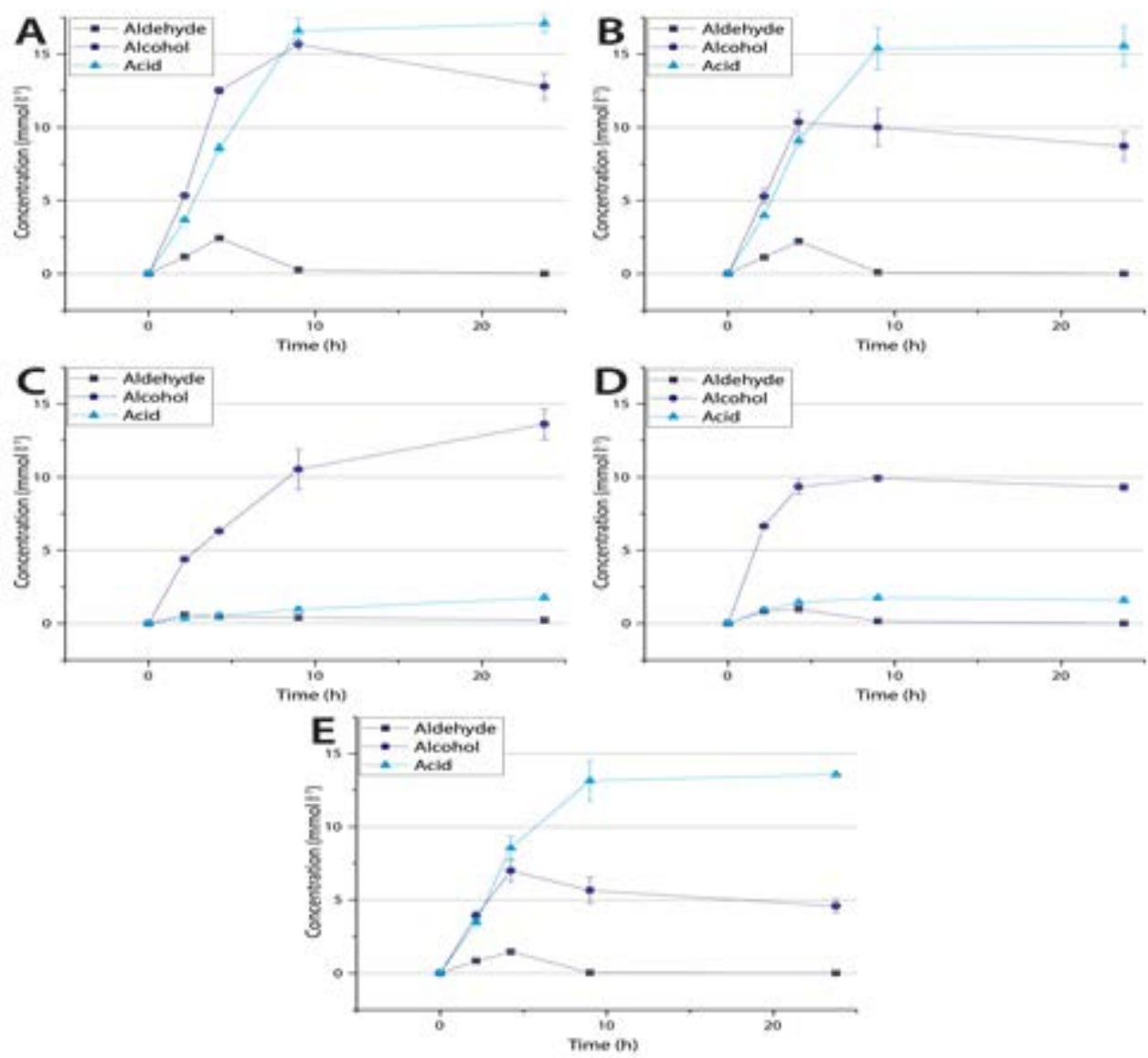

This article is protected by copyright. All rights reserved. 
Figure 3: PLS model M2 score scatter plot of response data showing X-scores (t) of the first component along the $\mathrm{x}$-axis and $\mathrm{X}$-scores of the second component along the $\mathrm{y}$-axis (A); loading scatter plot of the relation between factors and responses and the contribution of each variable to the PLS model; axes show weights for the factors and responses, denoted $\mathrm{w}^{*}$ and $\mathrm{c}$, respectively, for each model component (B).
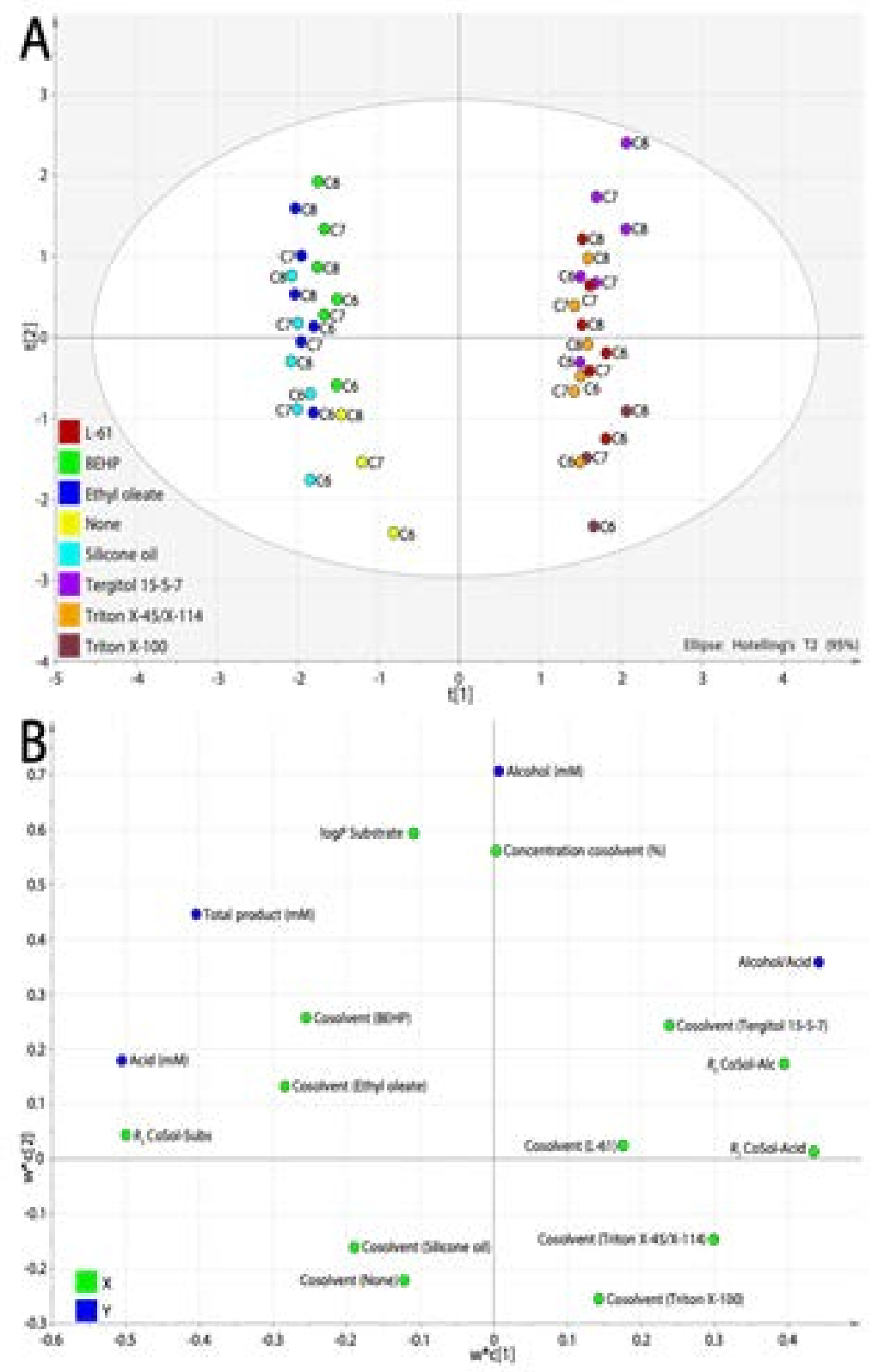

This article is protected by copyright. All rights reserved. 